\title{
Increased hippocampal TrkA expression ameliorates cranial radiation-induced neurogenesis impairment and cognitive deficit via PI3K/AKT signaling
}

\author{
SHENGJUN JI ${ }^{1-3}$, HAOHAO WU ${ }^{4}, \mathrm{XIN}_{\mathrm{DING}}{ }^{5}$, QINGQING CHEN ${ }^{3}, \mathrm{XING} \mathrm{JIN}^{3}$, JINMING YU ${ }^{1}$ and MING YANG ${ }^{2}$ \\ ${ }^{1}$ Clinical College, School of Medicine, Shandong University, Jinan, Shandong 250012; \\ ${ }^{2}$ Shandong Academy of Medical Sciences, Shandong First Medical University, Jinan, Shandong 250117; \\ ${ }^{3}$ Department of Radiotherapy and Oncology, The Affiliated Suzhou Hospital of Nanjing Medical University, \\ Suzhou, Jiangsu 215001; ${ }^{4}$ Department of Radiotherapy and Oncology, Yancheng No. 1 People's Hospital, \\ Yancheng, Jiangsu 224000; ${ }^{5}$ Department of Radiotherapy and Oncology, \\ The Affiliated Hospital of Xuzhou Medical University, Xuzhou, Jiangsu 221000, P.R. China
}

Received April 12, 2020; Accepted September 1, 2020

DOI: $10.3892 /$ or.2020.7782

\begin{abstract}
Cognitive deficit is one of the most serious complications of cranial radiotherapy of head and neck cancers. However, the underlying mechanism of this cognitive impairment remains unclear. In the present study, the role of tropomyosin receptor kinase A (TrkA) and its ligand neurotrophin nerve growth factor (NGF) were investigated following whole-brain irradiation (WBI). Young male Sprague-Dawley rats underwent WBI at a single dose of $10 \mathrm{~Gy}$. WBI was determined to result in notable memory decline and substantial neurogenesis impairment in the hippocampus 3 months post-irradiation. Compared with the control group, TrkA protein expression was greater in irradiated rats 1 week after WBI, which then decreased significantly by the 3-month time-point. However, no difference in NGF expression was observed from 1 day to 3 months post-WBI. Overexpression of hippocampal TrkA in rats using adeno-associated virus ameliorated memory
\end{abstract}

Correspondence to: Professor Jinming Yu, Clinical College, School of Medicine, Shandong University, 440 Jiyan Road, Jinan, Shandong 250012, P.R. China

E-mail: yujinming59@163.com

Professor Ming Yang, Shandong Academy of Medical Sciences, Shandong First Medical University, 18877 Jingshi Road, Jinan, Shandong 250117, P.R. China

E-mail: dryangming@sina.com

Abbreviations: NGF, nerve growth factor; TrkA, tropomyosin receptor kinase $\mathrm{A}$; WBI, whole-brain irradiation; ERK, extracellular signal-regulated protein kinase; CREB, cyclic adenosine monophosphate response element-binding protein; AAV, adeno-associated virus; $775^{\mathrm{NTR}}$, p75 neurotrophin receptor; GFP, green fluorescent protein; BrdU, 5-bromodeoxyuridine

Key words: cranial radiotherapy, hippocampus, memory, tropomyosin receptor kinase A decline induced by irradiation. Additionally, upregulating TrkA expression rescued irradiation-induced hippocampal precursor cell proliferation and promoted neurogenesis. PI3K, Akt and ERK1/2 phosphorylation were also revealed to be significantly inhibited by WBI, which was ameliorated by TrkA overexpression. Findings of the present study indicated that the TrkA-dependent signaling pathway may serve a critical role in radiotherapy-induced cognitive deficit and impairments in neurogenesis.

\section{Introduction}

Radiotherapy has been demonstrated to provide long-term survival benefits for patients with primary or secondary brain tumors (1-3). However, cognitive dysfunction following cranial irradiation is the most serious complication in these patients (4). The main clinical manifestations of this is the progressive reduction in hippocampal-dependent learning and memory and capacity to process information $(5,6)$. These delayed and long-term adverse effects can have a serious impact on the quality of life of the patient. Therefore, the neurocognitive state following cranial irradiation has been recommended by the Neurological Therapeutic Response Assessment Working Group to be one of the primary endpoints of clinical trials investigating brain tumors (7). Unfortunately, alternative effective treatment and predictive strategies for patients with brain tumors remain limited. In addition, a thorough understanding of the molecular events underlying irradiation-induced memory decline is required to identify novel therapeutic strategies.

Numerous studies have previously indicated that neurogenesis impairment in the hippocampus is critical for irradiation-induced cognitive deficit $(8,9)$. There are two main areas in the brain where neurogenesis primarily occurs: i) The dentate gyrus of the hippocampus; and ii) the subventricular area of the olfactory bulb. These new neurons migrate to the specific brain regions and incorporate into existing neural networks involved in mediating normal brain function $(10,11)$. 
Nerve growth factor (NGF) is a neurotrophic receptor that is highly expressed in the hippocampus, cortex and pituitary gland (12). NGF has been demonstrated to bind tropomyosin receptor kinase A (TrkA) and serve a key role in the development and functional maintenance of the brain $(13,14)$. Although NGF can also bind to the 75 neurotrophin receptor (p75 ${ }^{\mathrm{NTR}}$ ) at a lower affinity compared with TrkA, it is improbable that $\mathrm{p} 75^{\mathrm{NTR}}$ would preferentially transmit death signals in cells expressing both receptors $(14,15)$. The binding of NGF to TrkA activates the extracellular signal-regulated protein kinase (ERK)1/2 signaling pathway, which in turn activates the cyclic adenosine monophosphate response element-binding protein (CREB). Activation of CREB regulates various downstream signaling pathways required for neural precursor cell proliferation, survival and memory formation $(14,16)$.

However, the role of NGF-TrkA signaling in irradiation-induced memory deficit has not been fully determined. Therefore, in the present study, a rat model was used to investigate the influence of irradiation on NGF, TrkA and their associated downstream signaling events, in addition to their subsequent effects on hippocampal neurogenesis and memory deficit.

\section{Materials and methods}

Animals and whole-brain irradiation (WBI). The present study was approved by the Animal Care and Ethics Committee of The Affiliated Suzhou Hospital of Nanjing Medical University and Shandong University (Jinan, China). A total of 36 male Sprague-Dawley rats (weight, 50-60 g; age, 21 days) were obtained from The School of Medicine, Shandong University. All the rats were housed with 3-4 animals per cage with ad libitum access to tap water and food. The temperature was set at $24^{\circ} \mathrm{C}$ and the relative humidity was $50-70 \%$. They were kept under natural light in 12-h light/dark cycles. All rats were anesthetized with $4 \%$ isoflurane and placed in prone position in a 23EX linear accelerator (Varian Medical Systems, Inc.). A total of $1-2 \%$ isoflurane with $0.6-0.8 \mathrm{l} / \mathrm{min}$ flow rate maintained anesthesia during this process. Each rat was treated with WBI using a 4-MeV electron beam at a single dose of 10 or $0 \mathrm{~Gy}$ for the control group. The beam was directed downwards towards the head, whilst the body was shielded using a customized block.

Study design. After WBI, each rat received a twice daily intraperitoneal injection of bromo-deoxyuridine (BrdU; $50 \mathrm{mg} / \mathrm{kg}$ body weight; Sigma-Aldrich; Merck KGaA) for 4 days prior to irradiation exposure. All rats were anesthetized with $4 \%$ isoflurane followed by $1-2 \%$ isoflurane with $0.6-0.8 \mathrm{l} / \mathrm{min}$ flow rate to maintain anesthesia. Adeno-associated virus (serotype 8, AAV8) encoding GFP was stereotaxically infused into the dorsal hippocampus within $24 \mathrm{~h}$ after WBI. All Sprague-Dawley rats were randomly apportioned into the following groups ( $n=12$ per group): i) AAV-control, consisting of control rats infused with AAV encoding TrkA scramble sequence; ii) AAV-irradiation, consisting of irradiated rats infused with AAV encoding TrkA scramble sequence; and iii) AAV-overexpression (ovp)-TrkA, consisting of irradiated rats infused within AAV encoding overexpression TrkA. Morris water maze and open field test was performed at
1 and 3 months, following which all rats were sacrificed with carbon dioxide. The animals were placed into a box and $\mathrm{CO}_{2}$ was infused into the box at a rate of $10-30 \%$ of the volume of the euthanasia box every minute. After $5 \mathrm{~min}$, the animals exhibited no movements, no breathing, no heartbeat, and pupil dilation. Then, the infusion of $\mathrm{CO}_{2}$ was terminated and the rats were observed for another 2 min to confirm the death of the animal. Thus, a total of 7 min observation was used to confirm animal sacrifice. All the euthanasia procedures on the use of experimental animals adhered to the AVMA Guidelines for the Euthanasia of Animals (17) and the best efforts were made to minimize animal suffering throughout the experiment. The humane endpoints of this study included rats quickly losing $20 \%$ of their original body weight and rats that were unable to eat and drink on their own due to weakness. Immunofluorescence staining or western blotting were performed to evaluate the effects of irradiation on neurogenesis and protein expression.

BrdU labeling. Each rat received an intraperitoneal injection of $\mathrm{BrdU}$ ( $50 \mathrm{mg} / \mathrm{kg}$ body weight), twice daily at 8-h intervals, for a total period of 4 days before WBI.

$A A V$ and stereotaxic injection. An AAV serotype 8 encoding GFP vector was used in the present study. AAV8 encoding TrkA scramble sequence and overexpressing TrkA were constructed by Jikai Biological Technology Co., Ltd. AAV8 expressing a TrkA scramble sequence served as control. After anesthesia, rats were bilaterally infused with AAV8 into the dorsal hippocampus as previously described (18). Briefly, the injection site was $-3.7 \mathrm{~mm}$ anteroposterior from the bregma, $\pm 2.2 \mathrm{~mm}$ mediolateral from the bregma and $3.5 \mathrm{~mm}$ below the surface of the skull. The titer of AAV8 virus was $3.92 \times 10^{12} \mathrm{v} . \mathrm{g} . / \mathrm{ml}$ (vector genomes $/ \mathrm{ml}$ ), where a total volume of $2 \mu \mathrm{l}$ was injected. The rate of infusion was $0.2 \mu \mathrm{l} / \mathrm{min}$, where the cannula was left in place for $5 \mathrm{~min}$ to ensure the complete diffusion of the virus into the brain.

Morris water maze test. Place navigation tests were conducted on days 1-5, where all rats underwent 4 trials per day. A square platform, $\sim 9 \mathrm{~cm}$ in diameter, was submerged $1.5 \mathrm{~cm}$ beneath the water. The location of the platform remained constant throughout the trials, but the starting location changed for each trial. Each rat was given $60 \mathrm{sec}$ to locate the platform, where it was then permitted to rest for a further $10 \mathrm{sec}$ before being assisted back into the home cage. If the rat failed to locate the platform in the allotted time, it was guided to the platform and allowed to remain on it for $10 \mathrm{sec}$.

A spatial probe test took place on day 6, where the platform was removed and the rats were allowed to swim freely for $60 \mathrm{sec}$. The test was performed within $24 \mathrm{~h}$ after the trials for 4 days. The time taken to reach the platform, path length, swimming speed and the number of times the target zone was crossed were recorded.

Open field test. Using an opaque open field (410x410x505 mm), the middle and inner areas were termed the central region. Each rat was placed in the central region, and was allowed to move freely around the open field for $10 \mathrm{~min}$. An automated video-tracking system (Shanghai Jiliang Software Technology Co., Ltd.) was used to assess voluntary locomotor activity. 
Total track length, recorded as the total distance traveled and the time spent in the central region, were measured using the automated video-tracking system.

Western blotting analysis. Immediately after the behavior experiment, the experimental animals were sacrificed with carbon dioxide. The hippocampus tissue homogenates were lysed in RIPA buffer (cat. no. P0013B; Beyotime Institute of Biotechnology) and subjected to $10 \%$ SDS-PAGE (cat. no. ST628; Beyotime Institute of Biotechnology). Protein concentrations were determined using the bicinchoninic acid protein assay kit (cat. no. P0009; Beyotime Institute of Biotechnology). The polyvinylidene difluoride membranes were blocked in 5\% milk in TBST for $1 \mathrm{~h}$ at room temperature, incubated with primary antibodies overnight at $4^{\circ} \mathrm{C}$, and then incubated with secondary antibodies for $1 \mathrm{~h}$ at room temperature. The following antibodies were used: Anti-NGF (1:1,000; product code ab6199; Abcam), anti-TrkA (1:2,000; product code ab76291; Abcam), anti-phosphorylated (p)-PI3K (1:1,000; cat. no. 511120; ZenBioscience), anti-p-AKT (1:1,000; cat. no. 310022, ZenBioscience) and anti-p-Erk1/2 (1:1,000; cat. no. 310064; ZenBioscience). Anti-GAPDH (1:5,000; cat. no. ABS16), anti- $\beta$-actin $(1: 10,000$; cat. no. AV40173) and anti-tubulin (1:10,000; cat. no. T3526) were purchased from Sigma-Aldrich, Merck KGaA. Goat anti-mouse HRP (1:10,000; code no. 115-035-166) and goat anti-rabbit HRP (1:10,000; code no. 111-035-003) were purchased from Jackson ImmunoResearch, Laboratories Inc. Immunolabeling of membranes was detected by ECL chemiluminescence (P0018S; Beyotime Institute of Biotechnology). The integrated densities of each band were quantified using ImageJ Software (version 2006.02.01; National Institutes of Health).

Immunohistochemistry. Immediately after the behavior experiment, the rats were anesthetized and perfused with ice-cold saline followed by ice-cold $4 \%$ paraformaldehyde. Brain tissues were then removed and post-fixed overnight in $4 \%$ paraformaldehyde at $4{ }^{\circ} \mathrm{C}$ followed by equilibration in $30 \%$ sucrose. Sagittal sections at $30-\mu \mathrm{m}$-thick were cut at $-20^{\circ} \mathrm{C}$ by frozen slicer (Leica CM1950; Leica Microsystems, Inc.). The sections were treated with $2 \mathrm{M} \mathrm{HCl}$ at $37^{\circ} \mathrm{C}$ for half an hour and then washed in $1 \mathrm{X}$ Tris-buffered saline $\mathrm{pH} 8.5$ before incubation in 5\% BSA (cat. no. ST023; Beyotime Institute of Biotechnology) and 5\% Triton X-100 (Beyotime Institute of Biotechnology) in PBS for $1 \mathrm{~h}$ at room temperature. Tissue sections were incubated with rabbit anti-doublecortin (DCX; 1:100; cat. no. D9818; Sigma-Aldrich; Merck KGaA), mouse anti-neuronal nuclei (NeuN; 1:50; cat. no. MAB377; Thermo Fisher Scientific, Inc.), rabbit anti-glial cell marker glial fibrillary acidic protein (GFAP; 1:100; cat. no. ab207165, Abcam) or rabbit anti-BrdU (1:500; cat. no. B8434; EMD Millipore) primary antibodies at $4^{\circ} \mathrm{C}$ overnight, followed by a fluorescent secondary antibody conjugated with Alexa Fluor ${ }^{\circledR} 488$ goat anti-mouse (1:500; cat. no. A11001; Invitrogen; Thermo Fisher Scientific, Inc.) or Cy3-conjugated goat anti-rabbit (1:500; cat. no. 111-165-144; Jackson ImmunoResearch Laboratories, Inc.) secondary antibodies for $2 \mathrm{~h}$ at room temperature. Cell nuclei were stained with $100 \mathrm{ng} / \mathrm{ml}$ DAPI (cat. no. C1002; Beyotime Institute of Biotechnology) for $10 \mathrm{~min}$ at room temperature.
A confocal laser-scanning microscope (Olympus Corporation) with $\mathrm{x} 20$ objective was used to analyze the tissue staining. Cell counts were evaluated in 5-10 tissue sections per rat using a multi-channel configuration with ImageJ software (version 2006.02.01). Cell counts were limited to regions in the granule cell layer and hilus. The volumes of the granule cell layer and hilus were used to normalize the numbers of positive cells.

Statistical analysis. SPSS 22.0 software (IBM Corp.) was used to analyze all statistical data, and the results are expressed as the mean \pm standard error of the mean (SEM). Data were analyzed using unpaired t-test, one-way analysis of variance (ANOVA), or two-way ANOVA. Student-Newman-Keuls was used as the post hoc test following ANOVA. Mann-Whitney U-test was used to analyze non-normally distributed behavior test data. $\mathrm{P}<0.05$ was considered to indicate a statistically significant difference.

\section{Results}

Cranial irradiation induces spatial memory dysfunction. The effects of radiation on spatial learning and memory were investigated using spatial navigation tests 3 months after WBI. In the place navigation test, all rats displayed improved performance during days 1-4. On days 4 and 5, the irradiated rats exhibited significantly increased latency times before locating the submerged platform, compared with those of the non-irradiated rats $(\mathrm{P}<0.05$; Fig. 1A). Furthermore, in the spatial probe test, the irradiated rats spent significantly less time in the target quadrant compared with that of the non-irradiated rats $(\mathrm{P}<0.01$; Fig. $1 \mathrm{~B}$ and $\mathrm{C})$. These results demonstrated that irradiation was associated with spatial memory dysfunction.

An open field test was subsequently applied to assess the anxiety levels and spontaneous movement. The results revealed no significant differences in spontaneous movement or anxiety-like behavior between the two groups (Fig. 1D and E), which eliminates the influences of exercise and emotion on spatial memory dysfunction.

Cranial irradiation inhibits cellular proliferation and neurogenesis in the hippocampus. The results of the BrdU-labeling assay revealed that irradiated rats exhibited 74.2 and $73.9 \%$ fewer proliferating cells $(\mathrm{P}<0.01$; Fig. $2 \mathrm{~A}$ and $\mathrm{B})$ compared with those in non-irradiated rats after 1 and 3 months, respectively. Immature neurons were labeled with BrdU and DCX, a microtubule-associated protein expressed in migrating neuroblasts (19). The results revealed an 85.4 and $73.7 \%$ decrease in the number of BrdU- and DCX-positive cells after cranial irradiation $(\mathrm{P}<0.01$; Fig. $2 \mathrm{C}$ and $\mathrm{D})$. In addition, double labeling of BrdU and the glial cell marker GFAP also revealed reduced staining (32.9 and 66.7\%) in irradiated rats compared with those in the non-irradiated rats at 1 and 3 months $(\mathrm{P}<0.01$; Fig. $2 \mathrm{E}$ and $\mathrm{F})$. Mature neurons that had undergone recent division were labeled with BrdU and NeuN $\left(\mathrm{BrdU}^{+} / \mathrm{NeuN}^{+}\right)$. The irradiated rats exhibited 68.5 and $88.6 \%$ fewer $\mathrm{BrdU}^{+} / \mathrm{NeuN}^{+}$cells compared with those in the non-irradiated rats at 1 and 3 months, respectively $(\mathrm{P}<0.01$; Fig. 2G and $\mathrm{H}$ ). These results indicated WBI inhibited 

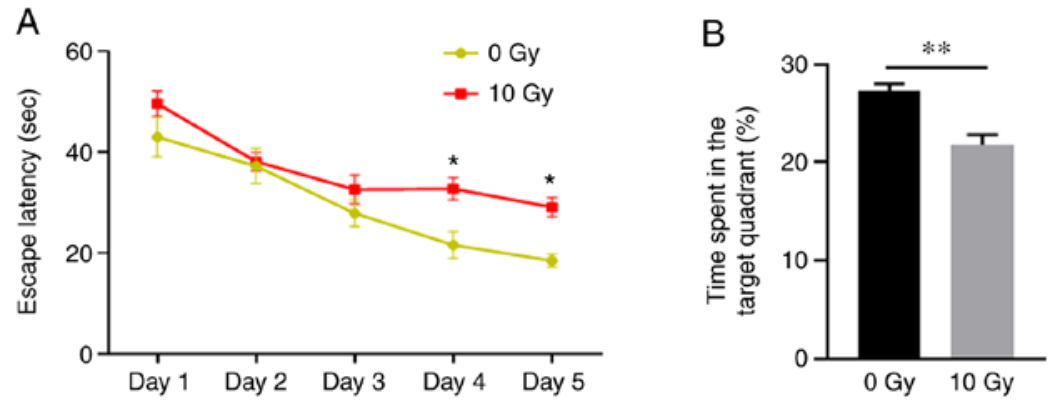

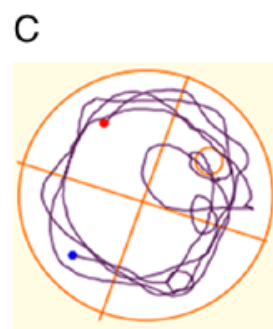

0 Gy

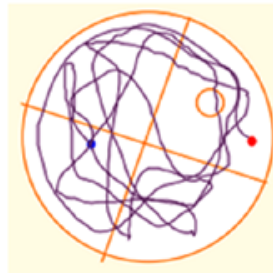

10 Gy
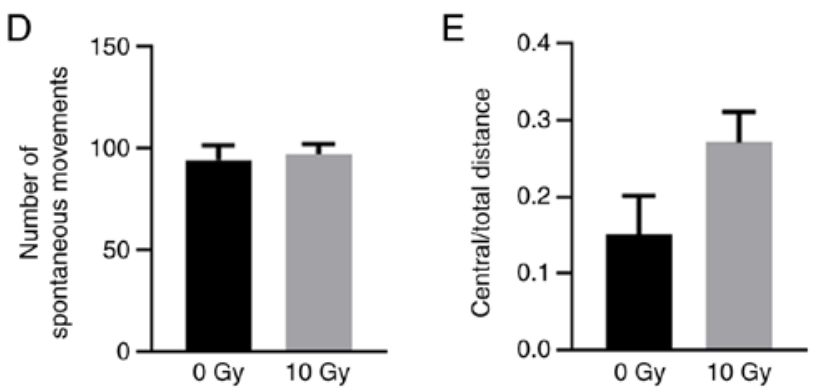

Figure 1. Behavioral testing following cranial irradiation. (A-C) Morris water maze test. (A) Escape latency and (B) percentage of target quadrant exploration time in the 0 and $10 \mathrm{~Gy}$ irradiation groups. (C) Representative images of swimming paths. (D and E) Open field test. Number of (D) spontaneous movements and (E) distance traveled in the central area recorded during 10 min of exposure to the training box. All data are presented as the mean $\pm \mathrm{SEM}$. ${ }^{*} \mathrm{P}<0.05$ and ${ }^{* *} \mathrm{P}<0.01$.

normal neuronal proliferation, differentiation, maturation and survival in the hippocampus.

Changes in hippocampal NGF and TrkA expression following cranial irradiation. Continuity detection was performed via western blotting, which was used to assess changes in the expression of NGF and TrkA in the rat hippocampus after irradiation. No differences in NGF protein expression were detected between irradiated and non-irradiated rats from 1 day to 3 months post-WBI (Fig. 3A and C). However, compared with that in the non-irradiated group, TrkA protein expression was found to be increased on days 1 and 3 , in addition to after 1 week $(\mathrm{P}<0.05)$, which was then decreased after the 1 -month time-point and was significantly reduced after 3 months $(\mathrm{P}<0.05$; Fig. 3B and $\mathrm{D})$.

TrkA overexpression prevents memory deficit in irradiated rats. The effects of TrkA overexpression on irradiation-induced memory deficit were subsequently investigated. AAV8s encoding TrkA scramble sequence and overexpressing TrkA (AAV-ovp-TrkA) were injected into the hippocampus of irradiated rats within $24 \mathrm{~h}$ after WBI (Fig. S1). In control rats, AAV8s encoding a TrkA scramble sequence were injected into irradiated rats (AAV-irradiation) and non-irradiated rats (AAV-ctl), respectively. Western blotting demonstrated the successful overexpression of TrkA in the hippocampus (Fig. S2). The influence of AAV8 injection on irradiated rats was also ruled out (Fig. S3). The levels of hippocampal TrkA expression in these three groups of rats were then detected by western blotting (Fig. 4A and B), which verified the effectiveness of AAV-ovp-TrkA infection. Morris water maze test was used to evaluate spatial memory function 3 months after WBI.

In the place navigation test, rats in the AAV-ovp-TrkA group exhibited shorter latency times prior to arrival at the hidden platform compared with those in the AAV-irradiation group on days 4 and 5 ( $\mathrm{P}<0.05$; Fig. $4 \mathrm{C})$. In the spatial probe trial, rats in the AAV-ovp-TrkA group also spent significantly more time in the target quadrant compared with those in the AAV-irradiation group ( $\mathrm{P}<0.05$; Fig. 4D). There was no statistical difference between the AAV-ctl and AAV-ovp-TrkA groups. The swim tracks revealed that rats in the AAV-irradiation group spent more time searching for the submerged platform compared with those in the other two groups (Fig. 4E).

$A A V$-induced TrkA overexpression in the irradiated rat hippocampus restores neurogenesis. At 3 months after WBI, intrahippocampal infusion of AAV-ovp-TrkA significantly prevented a reduction in neurogenesis when compared with rats in the AAV-irradiation group (Fig. 5A-D). The rats in AAV-ovp-TrkA group exhibited a $41.6 \%$ increase $\mathrm{BrdU}^{+} / \mathrm{NeuN}^{+}$ cells compared with those in the AAV-irradiation group $(\mathrm{P}<0.05$; Fig. 5D). These results indicated TrkA overexpression in the irradiated rat hippocampus restored neurogenesis.

Phosphorylation of PI3K, AKT and ERK1/2 is mediated by TrkA. The effect of cranial irradiation on the PI3K/AKT and ERK1/2 pathways was subsequently evaluated. The results revealed that the phosphorylation of PI3K, AKT and ERK1/2 was decreased following WBI (all $\mathrm{P}<0.05$; Fig. 6A and $\mathrm{B}$ ). However, tissues from the AAV-ovp-TrkA group exhibited increased PI3K, AKT and ERK1/2 phosphorylation compared with those in the AAV-irradiation group (Fig. 6A and B).

\section{Discussion}

Radiation cognitive impairment is a common side effect of radiotherapy for head and neck tumors, including glioma, nasopharyngeal carcinoma and brain metastatic tumors, 
A
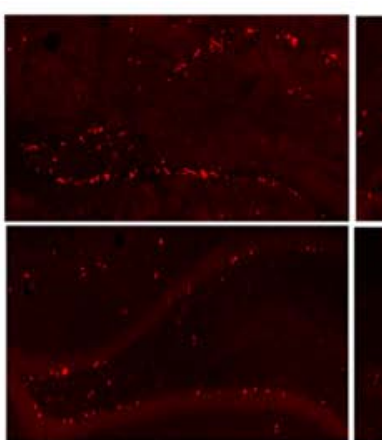

C

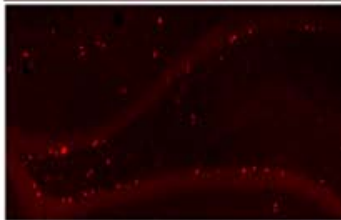

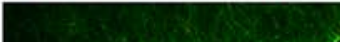
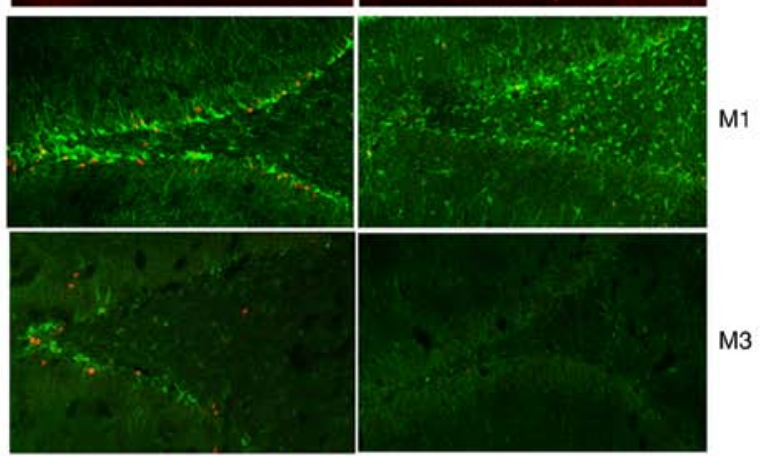

E
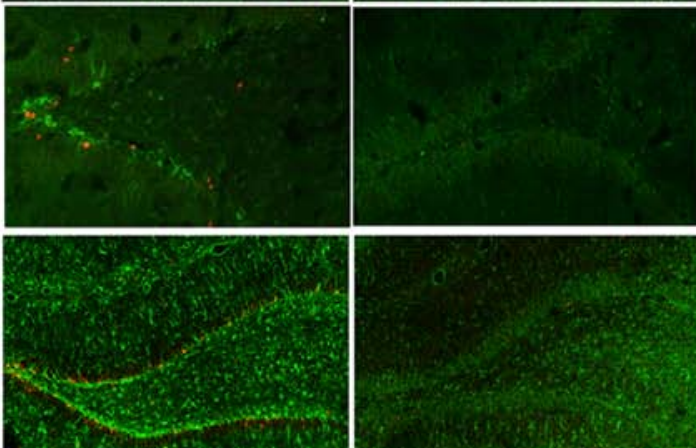

$\mathrm{M} 3$
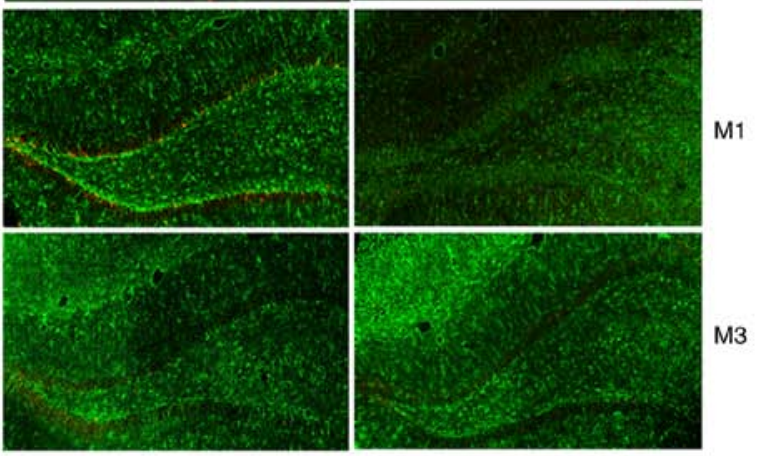

G

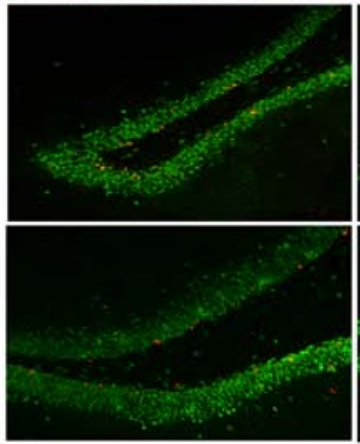

0 Gy

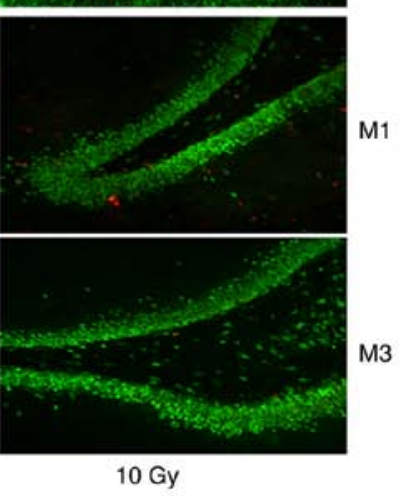

B

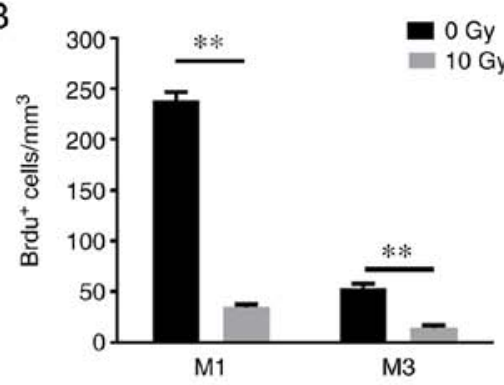

D

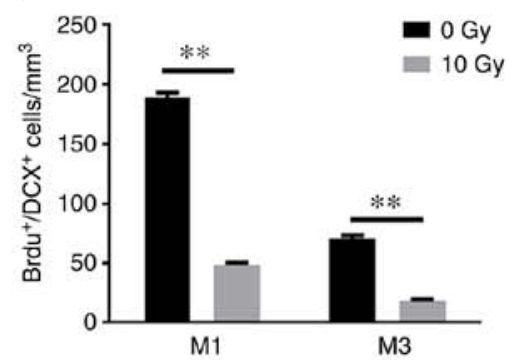

$\mathrm{F}$

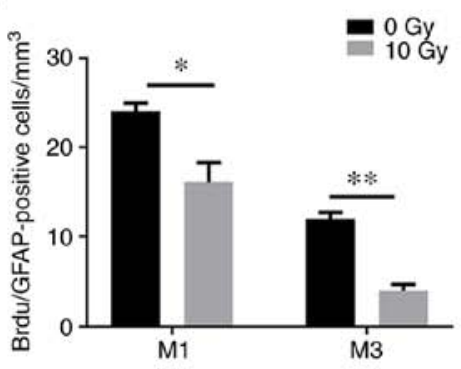

$\mathrm{H}$

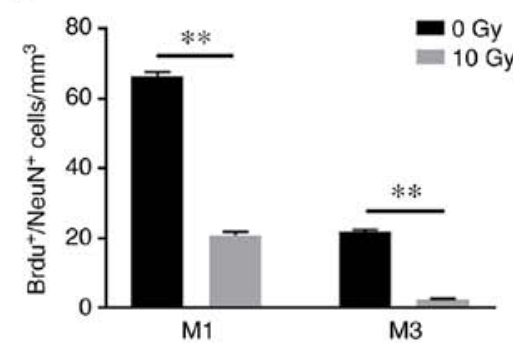

Figure 2. Cranial irradiation inhibits cellular proliferation and neurogenesis in the rat hippocampus. Confocal micrographs of hippocampal sections labeled with BrdU (red), DCX (green), GFAP (green) and NeuN (green). (A and B) Proliferating cells labeled with BrdU. (C and D) Immature neurons labeled with BrdU and DCX. (E and F) Differentiated astrocytes labeled with BrdU and GFAP. (G and H) Recently-divided mature neurons labeled with BrdU and NeuN. All histograms represent the mean \pm SEM. ${ }^{*} \mathrm{P}<0.05$ and ${ }^{* *} \mathrm{P}<0.01$. BrdU, 5-bromodeoxyuridine; DCX, doublecortin; GFAP, glial fibrillary acidic protein; NeuN, neuronal nuclei. Green: (C) DCX, (E) GFAP, and (G) NeuN; Red: BrdU.

which limits the efficacy of radiotherapy and damages the quality of life of patients (20). At present, it has attracted the attention of oncologists. The present study adds important clinical significance to the pathogenesis of radiation cognitive impairment.

Impairments in hippocampal neurogenesis have been demonstrated to serve a critical role in the progression of neurodegenerative diseases $(8,10,21)$. A number of studies have also indicated an association between neurogenesis impairment and memory decline in radiotherapy-treated animals $(8,9)$. Rola et al (9) first reported this phenomenon, showing a correlation between hippocampal neurogenesis and cognitive impairment after a single dose of WBI (5-10 Gy X-ray) in young mice. Since then, a series of studies have suggested that hippocampal neurogenesis impairment is significantly associated with the occurrence of cognitive defects $(8,22,23)$. However, the majority of these previous aforementioned studies only focused on the acute radiation response. 
A

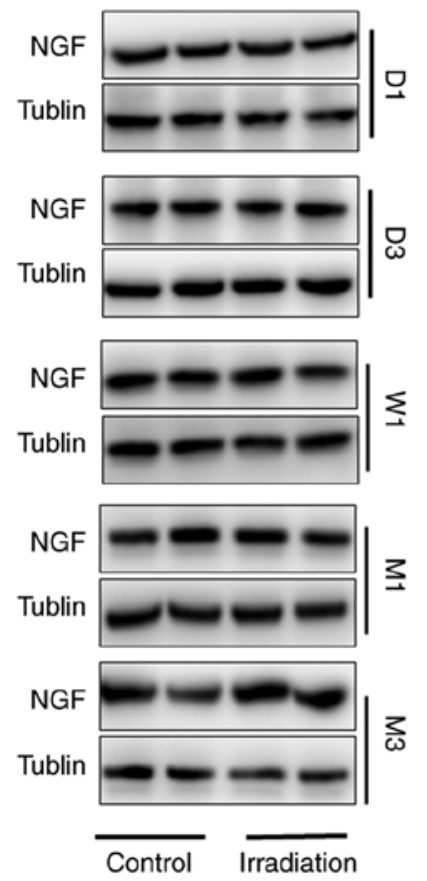

B

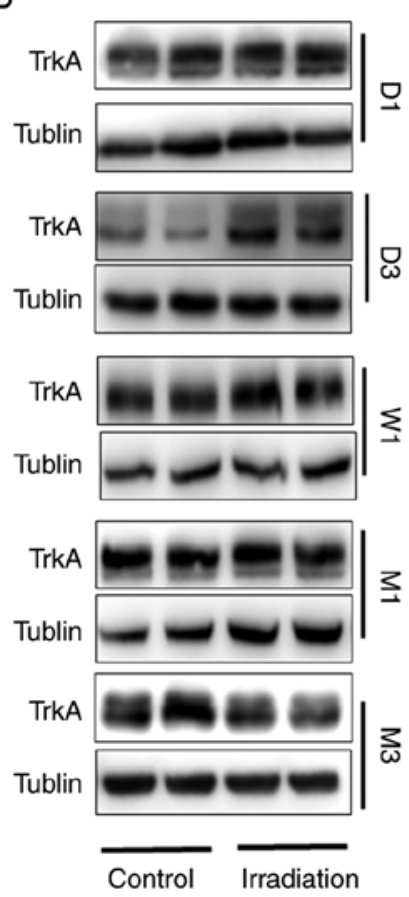

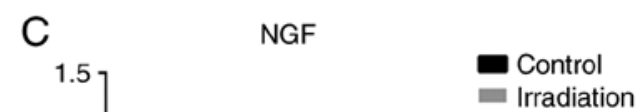

- Irradiation

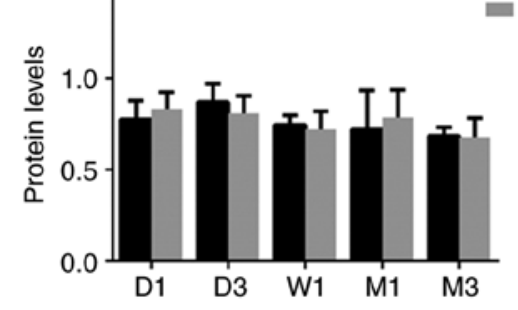

D
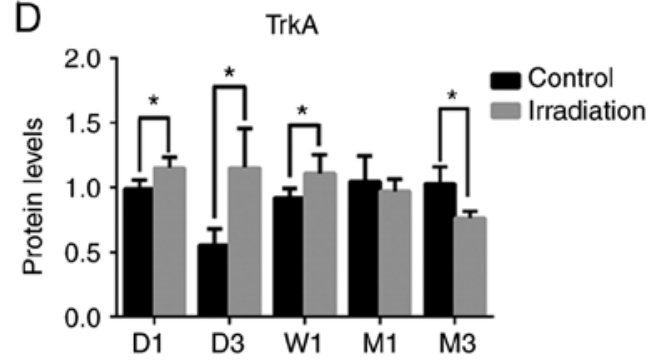

Figure 3. NGF and TrkA protein expression in the rat hippocampus following cranial irradiation. (A and C) NGF protein expression between irradiated and non-irradiated rats from 1 day to 3 months post-irradiation. (B and D) TrkA protein expression from 1 day to 3 months post-irradiation. All the images reveal non-adjacent bands from the same gel. Data are presented as the mean \pm SEM. ${ }^{*} \mathrm{P}<0.05$. NGF, nerve growth factor; TrkA, tropomyosin receptor kinase A.

Neural stem/progenitor cells are highly sensitive to irradiation in a dose-dependent manner $(8,9,24)$. Brain development in rats on postnatal day 21 is comparable to that of the preadolescent in humans (25). Because the rat brain is more resistant to radiation than the human brain, a 10-Gy dose in the rat approximates a clinically relevant dose in humans according to the Biological Effective Dose (BED) assuming an $\alpha / \beta$ ratio of 2 for late effects in normal brain tissue (26). Previous studies have found that 10 Gy WBI induced a decrease in neurogenesis in the rat hippocampus and resulted in spatial memory retention deficits after 3 months $(9,24,25,27)$. Therefore, in the present study, all rats received single WBI at $10 \mathrm{~Gy}$ to determine the persistence and severity of the relatively early effects on neurogenesis 3 months after irradiation. The results revealed that $10 \mathrm{~Gy}$ irradiation induced long-term and sustained impairments in cell proliferation as revealed by BrdU labeling, neuronal maturation as demonstrated by DCX labeling and neurogenesis as determined by BrdU and NeuN co-labeling. The Morris water maze test also revealed that WBI induced spatial memory deficits. These results indicated that cranial irradiation induces long-term impairment in hippocampal neurogenesis and spatial memory.

NGF is an important neurotrophin involved in the development of neural stem cells and synaptic plasticity $(12,14,28)$. In the adult brain, NGF also supports neural maintenance and repair (12). NGF binds to and activates specific receptor tyrosine kinases to facilitate neural stem cell differentiation. TrkA has been identified as the primary NGF receptor involved in both neuroprotection and neurodegenerative diseases $(16,29)$. Therefore, investigating changes in the expression of NGF and TrkA may provide a therapeutically beneficial target for radiotherapy-induced neurogenesis impairment and memory decline. NGF mainly binds to TrkA to activate downstream related signaling pathways. Conversely, p75NTR has only a low affinity (30). Thus, in the present study, the potential inhibitory effects of irradiation on NGF-TrkA signaling were investigated in hippocampal tissues. NGF expression levels were revealed to be similar between irradiated and non-irradiated rats from 1 day to 3 months post-WBI. This result is not consistent with those from studies of other neurodegenerative diseases $(12,14,16)$. However, in clinical application, the treatment strategy developed for neurotrophins, such as NGF and brain-derived neurotrophic factor, has not shown the desired effect $(28,31,32)$. Therefore, it was hypothesized that defects in the neurotrophins themselves may prevent their use as effective biotherapeutic agents. The therapeutic application of natural neurotrophins is also limited by poor oral bioavailability, poor permeability through the blood-brain barrier, undetermined pharmacokinetics and a short plasma half-life $(28,31,33)$. Notably, the expression of various specific neurotrophin receptors was revealed to be absent in patients with neurodegenerative and brain injury diseases, thereby limiting the biological roles of neurotrophins in a therapeutic setting $(16,34,35)$.

In the present study, the expression levels of TrkA were detected in the hippocampus following irradiation. Dynamic changes in TrkA expression were revealed, with TrkA protein expression upregulated during week 1, before gradually decreasing 1 and 3 months post-WBI. In several previous studies of neurodegenerative diseases, changes in TrkA receptor expression have been revealed to be closely associated with neurological function $(33,34,35)$. In addition, TrkA expression is closely related to neuronal survival and development. In a study conducted by Haque et al (36), calotropis 

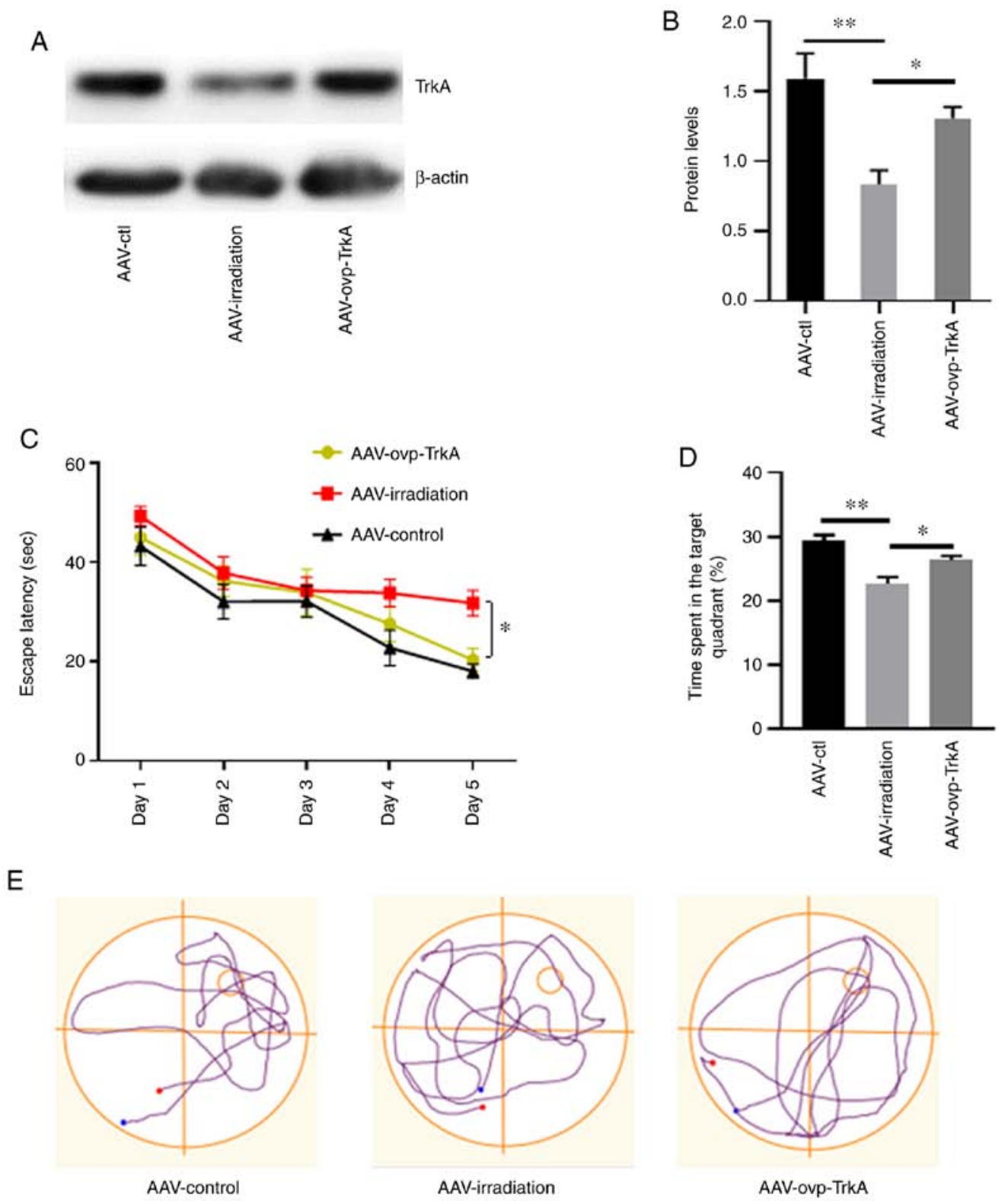

Figure 4. TrkA overexpression prevents memory deficit in the irradiated rat. (A and B) Levels of TrkA in hippocampal extracts from rats in the AAV-ctl, AAV-irradiation and AAV-ovp-TrkA groups following AVV administration. (C-E) Morris water maze test data. (C) Escape latencies and (D) percentage of target quadrant exploration time in the probe test among rats in the AAV-ctl, AAV-irradiation and AAV-ovp-TrkA groups. (E) Representative images of swimming paths. Data are presented as the mean \pm SEM. ${ }^{*} \mathrm{P}<0.05$ and ${ }^{* *} \mathrm{P}<0.01$. AAV, adeno-associated virus; ovp, overexpressing; TrkA, tropomyosin receptor kinase A; ctl, control.

gigantean was revealed to promote the neuritogenic and synaptogenic potential of hippocampus neurons via the activation of NGF-TrkA-Erk1/2 signaling, which was inhibited by a TrkA-specific inhibitor. Another previous study demonstrated that organophosphate pesticides reduced the survival time of basal forebrain cholinergic neurons by inhibiting TrkA (35). Results of the present study revealed that irradiation specifically decreased TrkA expression but that of NGF remained unchanged. This finding indicated that TrkA serves a prominent role in radiotherapy-induced neurogenesis impairment.

To verify the role of TrkA in radiotherapy-induced neurogenesis impairment and memory decline further, an AAV virus was constructed to overexpress TrkA. TrkA overexpression in the irradiated rat hippocampus was revealed to ameliorate spatial memory decline and prevent hippocampal neurogenesis impairment, which further supports the crucial protective role of TrkA.
NGF-TrkA interactions may activate multiple intracellular signaling pathways, such as the MAPK, PI3K-AKT and phospholipase $C \gamma$ cascades $(12,16,37)$. Furthermore, key signaling molecules downstream of TrkA were investigated, including those involved in cell survival and death. The present study demonstrated that 10 Gy WBI inhibited ERK1/2 and PI3K-Akt activation. Following the activation of TrkA, ERK1/2 was able to upregulate the transcription factor CREB, which regulates the transcription of various genes responsible for the maintenance of neurogenesis, plasticity and memory consolidation (38). AKT phosphorylation activated by TrkA is also considered to be one of the key factors in the regulation of cell survival and apoptosis (37).

In conclusion, results from the present study indicated a previously unreported radiation-induced memory deficit and neurogenesis impairment in a manner that is dependent on 

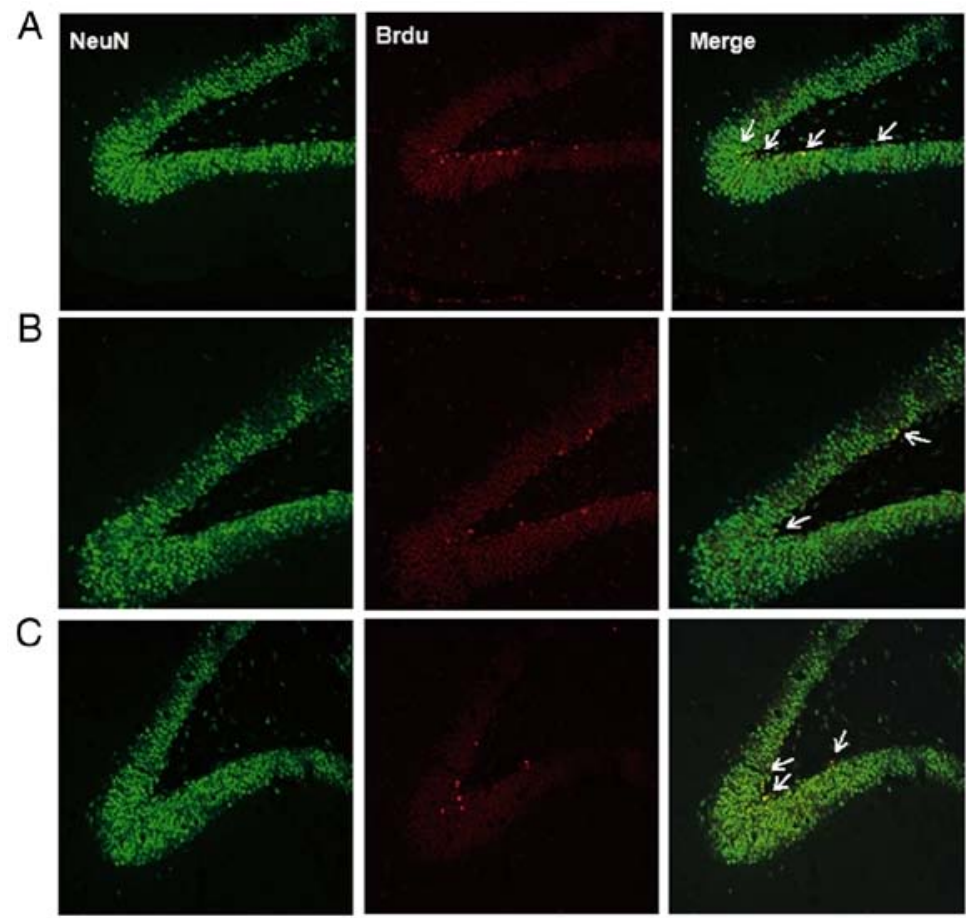

D

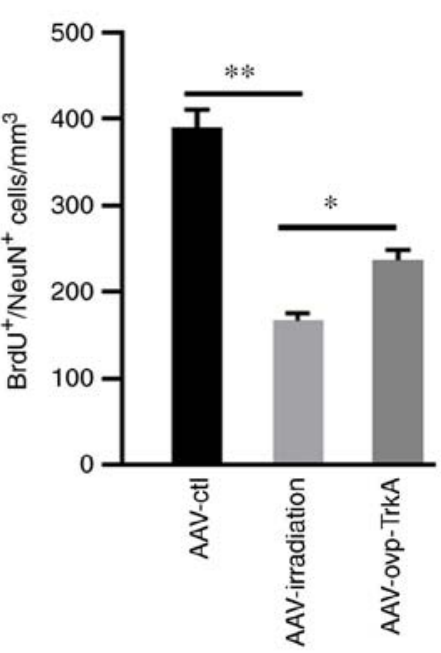

Figure 5. AAV-TrkA expression in the irradiated rat hippocampus prevents neurogenesis impairment. TrkA overexpression rescued irradiation-induced neurogenesis impairment. NeuN, BrdU and $\mathrm{NeuN}^{+} / \mathrm{BrdU}^{+}$double staining in (A) AAV-ctl, (B) AAV-irradiation and (C) AAV-ovp-TrkA groups. (D) Number of $\mathrm{NeuN}^{+} / \mathrm{BrdU}^{+}$cells in the AAV-ctl, AAV-irradiation and AAV-ovp-TrkA groups. Data are presented as the mean $\pm \mathrm{SEM}$. ${ }^{*} \mathrm{P}<0.05$ and ${ }^{* *} \mathrm{P}<0.01$. Green, NeuN; red, BrdU; yellow, $\mathrm{NeuN}^{+} / \mathrm{BrdU}^{+}$. BrdU, 5-bromodeoxyuridine; AAV, adeno-associated virus; TrkA, tropomyosin receptor kinase A; NeuN, neuronal nuclei; ctl, control; ovp, overexpressing.

A
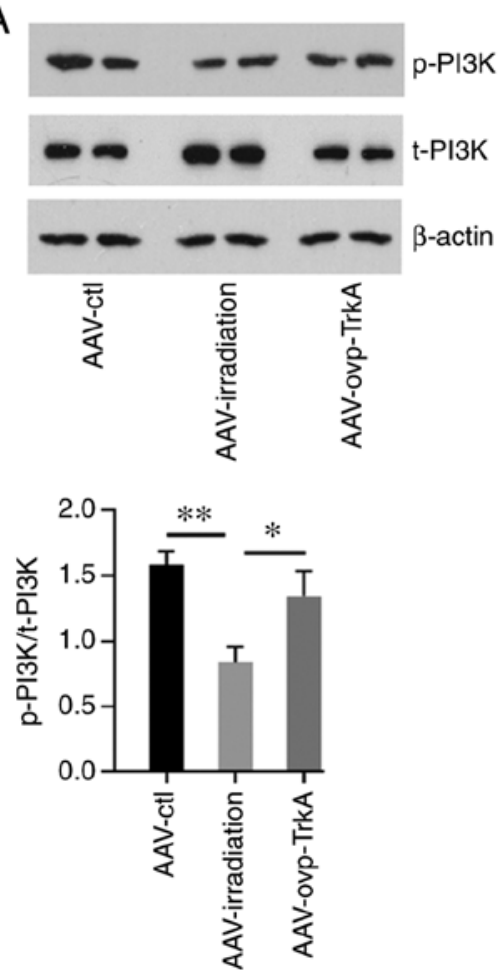

B
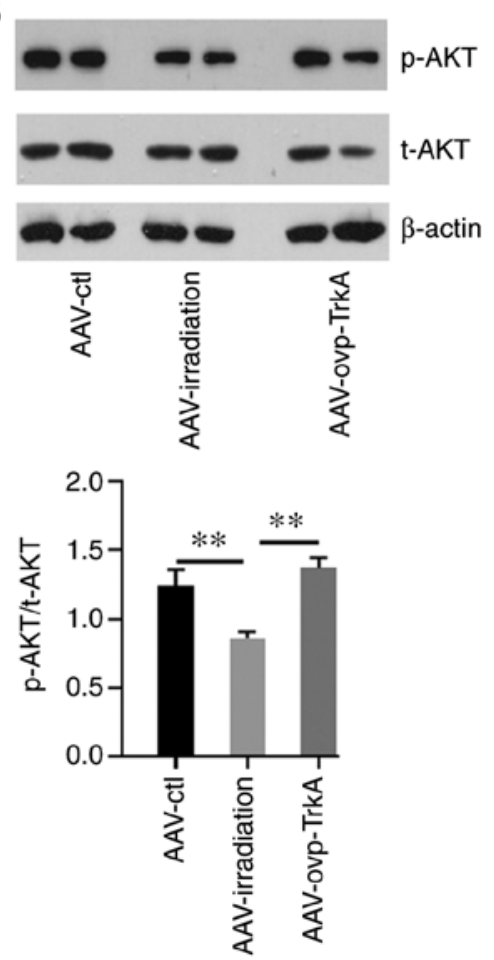

C
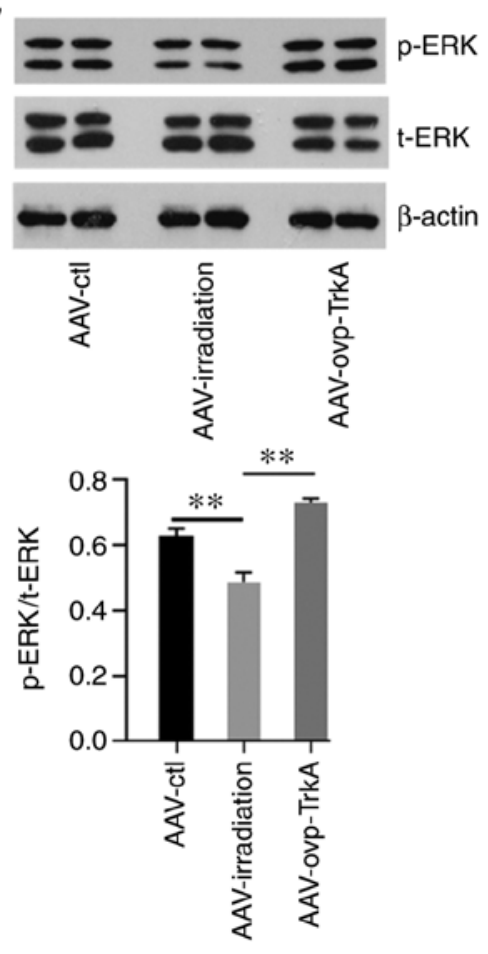

Figure 6. Downstream signaling molecules of TrkA after AAV-TrkA expression. PI3K (A), AKT (B) and ERK1/2 (C) phosphorylation in the AAV-ctl, AAV-irradiation and AAV-ovp-TrkA groups was examined. All the images reveal non-adjacent bands from the same gel. Data are presented as the mean \pm SEM. ${ }^{*}<0.05$ and ${ }^{* * *} \mathrm{P}<0.01$. TrkA, tropomyosin receptor kinase A; $\mathrm{p}-$, phosphorylated; AAV, adeno-associated virus; ctl, control; ovp, overexpressing.

TrkA-dependent signaling. Therefore, future development of TrkA-dependent signaling agonists may serve as a promising therapeutic approach for reducing radiation-induced adverse effects in patients with brain tumors. 


\section{Acknowledgements}

We would like to thank Dr Junjun Zhang and Dr Qixian Zhang at The Second Affiliated Hospital of Soochow University (Suzhou, China) for their technical assistance. We would like to thank Dr Rui Sun at The Third Affiliated Hospital of Soochow University (Changzhou, China) for manuscript revision.

\section{Funding}

The present study was supported by the Natural Science Foundation of Jiangsu Province (grantno.BK20171224), the China Postdoctoral Science Foundation (grant no. 2018M632683), the 'Six One Project' of Jiangsu Province (grant no. LGY2018009), the Youth Talent Project of Changzhou Health Commission (grant no. QN201804) and the Youth Medical Fund of Yancheng 1st People's Hospital (grant no. QN2018007).

\section{Availability of data and materials}

The data generated and analyzed during the present study are available from the corresponding author on reasonable request.

\section{Authors' contributions}

SJ, JY and MY designed the present study. SJ and HW carried out the experiments and wrote the manuscript. XD, QC and XJ coordinated the study and analyzed the data. SJ, JY and MY revised it critically for important intellectual content. All authors were involved in writing the paper and approved the final manuscript.

\section{Ethics approval and consent to participate}

The present study was approved by the Animal Care and Ethics Committee of The Affiliated Suzhou Hospital of Nanjing Medical University and Shandong University.

\section{Patient consent for publication}

Not applicable.

\section{Competing interests}

The authors declare that they have no competing interests.

\section{References}

1. Koyfman SA, Ismaila N, Crook D, D'Cruz A, Rodriguez CP, Sher DJ, Silbermins D, Sturgis EM, Tsue TT, Weiss J, et al: Management of the neck in squamous cell carcinoma of the oral cavity and oropharynx: ASCO Clinical Practice Guideline. J Clin Oncol 37: 1753-1774, 2019.

2. Caudell JJ, Torres-Roca JF, Gillies RJ, Enderling H, Kim S, Rishi A, Moros EG and Harrison LB: The future of personalised radiotherapy for head and neck cancer. Lancet Oncol 18 e266-e273, 2017.

3. Gillison ML, Trotti AM, Harris J, Eisbruch A, Harari PM, Adelstein DJ, Jordan RCK, Zhao W, Sturgis EM, Burtness B, et al: Radiotherapy plus cetuximab or cisplatin in human papillomavirus-positive oropharyngeal cancer (NRG Oncology RTOG 1016): A randomised, multicentre, non-inferiority trial. Lancet 393: 40-50, 2019.
4. Hansen CC, Chrane K, Gunn GB, Mohamed ASR, Rosenthal DI, Wefel JS, Phan J, Frank SJ, Garden AS, Smith B, et al: Cognitive function and patient-reported memory problem following radiation therapy for cancers at the skull base: A survivorship study using the telephone interview for cognitive status and the MDASI-HN. Int J Radiat Oncol 94: 967-967, 2016.

5. Wang M, Cairncross G, Shaw E, Jenkins R, Scheithauer B, Brachman D, Buckner J, Fink K, Souhami L, Laperriere N, et al: Cognition and quality of life after chemotherapy plus radiotherapy (RT) vs. RT for pure and mixed anaplastic oligodendrogliomas: Radiation therapy oncology group trial 9402. Int J Radiat Oncol 77: 662-669, 2010.

6. Cheung YT, Sabin ND, Reddick WE, Bhojwani D, Liu W, Brinkman TM, Glass JO, Hwang SN, Srivastava D, Pui $\mathrm{CH}$, et al: Leukoencephalopathy and long-term neurobehavioural, neurocognitive, and brain imaging outcomes in survivors of childhood acute lymphoblastic leukaemia treated with chemotherapy: A longitudinal analysis. Lancet Haematol 3: e456-e466, 2016.

7. Alexander BM, Brown PD, Ahluwalia MS, Aoyama H, Baumert BG, Chang SM, Gaspar LE, Kalkanis SN, Macdonald DR, Mehta MP, et al: Clinical trial design for local therapies for brain metastases: A guideline by the Response Assessment in Neuro-Oncology Brain Metastases working group. Lancet Oncol 19: e33-e42, 2018.

8. Ji S, Tian Y, Lu Y, Sun R, Ji J, Zhang L and Duan S: Irradiation-induced hippocampal neurogenesis impairment is associated with epigenetic regulation of bdnf gene transcription. Brain Res 1577: 77-88, 2014.

9. Rola R, Raber J, Rizk A, Otsuka S, VandenBerg SR, Morhardt DR and Fike JR: Radiation-induced impairment of hippocampal neurogenesis is associated with cognitive deficits in young mice. Exp Neurol 188: 316-330, 2004.

10. Snyder JS: Recalibrating the relevance of adult neurogenesis. Trends Neurosci 42: 164-178, 2019.

11. Genin EC, Caron N, Vandenbosch R, Nguyen L and Malgrange B: Concise review: Forkhead pathway in the control of adult neurogenesis. Stem Cells 32: 1398-1407, 2014.

12. Aloe L, Rocco ML, Bianchi P and Manni L: Nerve growth factor: From the early discoveries to the potential clinical use. J Trans Med 10: 239, 2012.

13. Troy CM, Friedman JE and Friedman WJ: Mechanisms of p75-mediated death of hippocampal neurons. Role of caspases. J Bio Chem 277: 34295-34302, 2002.

14. Pramanik S, Sulistio YA and Heese K: Neurotrophin signaling and stem cells-implications for neurodegenerative diseases and stem cell therapy. Mol Neurobiol 54: 7401-7459, 2017.

15. Donnelly CR, Gabreski NA, Suh EB, Chowdhury M and Pierchala BA: Non-canonical Ret signaling augments p75-mediated cell death in developing sympathetic neurons. J Cell Biol 217: 3237-3253, 2018.

16. Sang Q, Sun D, Chen Z and Zhao W: NGF and PI3K/Akt signaling participate in the ventral motor neuronal protection of curcumin in sciatic nerve injury rat models. Biomed Pharmacother 103: 1146-1153, 2018.

17. AVMA Guidelines for the Euthanasia of Animals: 2020 Edition. Accessed from: https://www.avma.org/resources-tools/avma-policies/avma-guidelines-euthanasia-animals.

18. Ding X, Wu HH, Ji SJ, Cai S, Dai PW, Xu ML, Zhang JJ, Zhang QX, Tian Y and Ma QH: The p75 neurotrophin receptor regulates cranial irradiation-induced hippocampus-dependent cognitive dysfunction. Oncotarget 8: 40544-40557, 2017.

19. Katharina M and Chichung LD: Evidence that Doublecortin is dispensable for the development of adult born neurons in mice. PLoS One 8: e62693, 2013.

20. Soussain C, Ricard D, Fike JR, Mazeron JJ, Psimaras D and Delattre JY: CNS complications of radiotherapy and chemotherapy. Lancet 374: 1639-1651, 2009.

21. Bao $\mathrm{H}$ and Song J: Treating brain disorders by targeting adult neural stem cells. Trends Mol Med 24: 991-1006, 2018.

22. Kitamura T, Saitoh Y, Takashima N, Murayama A, Niibori Y, Ageta H, Sekiguchi M, Sugiyama H and Inokuchi K: Adult neurogenesis modulates the hippocampus-dependent period of associative fear memory. Cell 139: 814-827, 2009.

23. Alam MJ, Kitamura T, Saitoh Y, Ohkawa N, Kondo T and Inokuchi K: Adult neurogenesis conserves hippocampal memory capacity. J Neurosci 38: 6854-6863, 2018.

24. Monje MJ, Mizumatsu S, Fike JR and Palmer TD: Irradiation induces neural precursor-cell dysfunction. Nat Med 8: 955-962, 2002. 
25. Achanta P, Fuss M and Martinez JL Jr: Ionizing radiation impairs the formation of trace fear memories and reduces hippocampal neurogenesis. Behav Neurosci 123: 1036-1045, 2009.

26. Sun R, Zhang LY, Chen LS and Tian Y: Long-term outcome of changes in cognitive function of young rats after various/different doses of whole brain irradiation. Neurol Res 38: 647-654, 2016.

27. Winocur G, Wojtowicz JM, Sekeres M, Snyder JS and Wang S: Inhibition of neurogenesis interferes with hippocampusdependent memory function. Hippocampus 16: 296-304, 2006.

28. Skup M: Neurotrophins: Evolution of concepts on rational therapeutic approaches. Postepy Biochem 64: 231-241, 2018

29. Khotskaya YB, Holla VR, Farago AF, Mills Shaw KR Meric-Bernstam F and Hong DS: Targeting TRK family proteins in cancer. Pharmacol Therap 173: 58-66, 2017.

30. Wehrman T, He X, Raab B, Dukipatti A, Blau H and Garcia KC: Structural and mechanistic insights into nerve growth factor interactions with the TrkA and p75 receptors. Neuron 53: 25-38, 2007.

31. Ochs G, Penn RD, York M, Giess R, Beck M, Tonn J, Haigh J, Malta E, Traub M, Sendtner M and Toyka KV: A phase I/II trial of recombinant methionyl human brain derived neurotrophic factor administered by intrathecal infusion to patients with amyotrophic lateral sclerosis. Amyotroph Lateral Scler Other Motor Neuron Disord 1: 201-206, 2000.

32. Mishra BR, Maiti R, Nath S, Sahoo P, Jena M and Mishra A: Effect of sertraline, dosulepin, and venlafaxine on non-BDNF neurotrophins in patients with depression: A cohort study. J Clin Psychopharmacol 39: 220-225, 2019.

33. Houlton J, Abumaria N, Hinkley SFR and Clarkson AN: Therapeutic potential of neurotrophins for repair after brain injury: A helping hand from biomaterials. Front Neurosci 13: 790, 2019.
34. Shah AG, Friedman MJ, Huang S, Roberts M, Li XJ and Li S: Transcriptional dysregulation of TrkA associates with neurodegeneration in spinocerebellar ataxia type 17. Hum Mol Genet 18: 4141-4152, 2009.

35. Kumar V, Gupta AK, Shukla RK, Tripathi VK, Jahan S, Pandey A, Srivastava A, Agrawal M, Yadav S, Khanna VK and Pant AB: Molecular mechanism of switching of TrkA/p75(NTR) signaling in monocrotophos induced neurotoxicity. Sci Rep 5: $14038,2015$.

36. Haque MN, Mohibbullah M, Hong YK and Moon IS: Calotropis gigantea promotes neuritogenesis and synaptogenesis through activation of NGF-TrkA-Erk1/2 signaling in rat hippocampal neurons. Am J Chin Med 46: 1861-1877, 2018.

37. Kim MS, Shutov LP, Gnanasekaran A, Lin Z, Rysted JE, Ulrich JD and Usachev YM: Nerve growth factor (NGF) regulates activity of nuclear factor of activated T-cells (NFAT) in neurons via the phosphatidylinositol 3-kinase (PI3K)-Akt-glycogen synthase

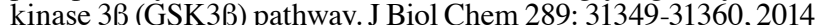

38. Arthur JS, Fong AL, Dwyer JM, Davare M, Reese E, Obrietan K and Impey S: Mitogen- and stress-activated protein kinase 1 mediates cAMP response element-binding protein phosphorylation and activation by neurotrophins. J Neurosci 24: 4324-4332, 2004.

This work is licensed under a Creative Commons Attribution-NonCommercial-NoDerivatives 4.0 International (CC BY-NC-ND 4.0) License. 